\title{
Folinic acid in colorectal cancer: esquire or fellow knight? Real- world results from a mono institutional, retrospective study
}

\author{
Francesco Jacopo Romano ${ }^{1}$, Carmela Barbato ${ }^{1}$, Maria Biglietto ${ }^{1}$, Vincenzo Di Lauro ${ }^{2}$, \\ Dario Arundine ${ }^{2}$, Roberto Fiorentino ${ }^{1}$, Francesca Ambrosio ${ }^{1}$, Maresa Cammarota ${ }^{3}$, \\ Bruno Chiurazzi ${ }^{1}$, Livio Puglia ${ }^{1}$, Sarah Scagliarini ${ }^{1}$, Raffaella Ruocco ${ }^{1}$, Carmen \\ Mocerino $^{1}$, Ivana Cerillo ${ }^{1}$, Maria Fiorella Brangi ${ }^{1}$ and Ferdinando Riccardi $^{1}$ \\ ${ }^{1}$ Antonio Cardarelli Hospital, Oncology Unit, Naples, Italy \\ ${ }^{2}$ Federico II University, Department of Medicine and Surgery, Oncology Unit, Naples, Italy \\ ${ }^{3}$ Antonio Cardarelli Hospital, Pharmacy Unit, Naples, Italy \\ Correspondence to: Francesco Jacopo Romano, email: francesco_jacopo@libero.it
}

Keywords: colorectal cancer; folinic acid; sodium levofolinate; thymidylate synthase; non oncogene addiction Received: September 28, $2020 \quad$ Accepted: January 07, $2021 \quad$ Published: February 02, 2021

Copyright: $\odot 2021$ Romano et al. This is an open access article distributed under the terms of the Creative Commons Attribution License (CC BY 3.0), which permits unrestricted use, distribution, and reproduction in any medium, provided the original author and source are credited.

ABSTRACT

The stock of therapeutic weapons available in metastatic colorectal cancer (mCRC) has been progressively grown over the years, with improving both survival and patients' clinical outcome: notwithstanding advances in the knowledge of mCRC biology, as well as advances in treatment, fluoropyrimidine antimetabolite drugs have been for 30 years the mainstay of chemotherapy protocols for this malignancy. 5-Fluorouracil (5FU) seems to act differently depending on administration method: elastomer-mediated continuous infusion better inhibits Thymidylate Synthase (TS), an enzyme playing a pivotal role in DNA synthetic pathway. TS overexpression is an acknowledged poor prognosis predicting factor. The simultaneous combination of $5 \mathrm{FU}$ and folinate salt synergistically strengthens fluorouracil cytotoxic effect. In our experience, levofolinate and 5FU together in continuous infusion prolong progression free survival of patients suffering from mCRC, moreover decreasing death risk and showing a clear clinical benefit for patients, irrespective of RAS mutational status, primitive tumor side and metastases surgery.

\section{INTRODUCTION}

Notwithstanding advances in the knowledge of metastatic colorectal cancer (mCRC) biology, as well as advances in treatment, fluoropyrimidine antimetabolite drugs (5-fluorouracil - FUra, 5FU, 5-fluoro-2'deoxyuridine - 5FUdR, 5'-deoxy-5-fluoro-N-[(pentyloxy) carbonyl]-cytidine - capecitabine, an oral FUra prodrug) are currently the mainstay of chemotherapy protocols for this malignancy. 5FU seems to act differently depending on administration method: quick bolus mainly increases incorporation of 5FU in RNA [1], even yielding a more severe hematological and gastrointestinal toxicity than continuous infusion [2], whereas elastomer-mediated continuous infusion long inhibits Thymidylate Synthase (TS) $[3,4]$. TS is an enzyme playing a pivotal role in DNA synthetic pathway by producing a crucial nucleotide for genome integrity and cellular economy: Colon Rectal Cancer (CRC) cells exhibit a large non-oncogene addiction toward TS, and its overexpression in CRC cells is an acknowledged poor prognosis predicting factor $[5,6]$.

Preclinical evidence has pointed out that culture medium containing folinic acid increases $5 \mathrm{FU}$-mediated cell growth inhibition and cytotoxicity (Figure 1). Actually, folinic acid acts as a "stabilizer" of fluoropyrimidines and TS, with accumulating inactive complexes [7,8]. Modulation of 5FU activity has been studied for several years, with the aim to enhance antineoplastic effect by combining bolus and continuous infusion administration to maximize 5FU antitumor efficacy. However, 5FU incorporation into RNA and DNA of tumor tissue seems to not correlate with treatment efficacy, unlike TS inhibition [9].

Therefore, therapeutic strategies combining bolus and continuous infusion have been made to better exploit both genotoxic effect of fluoropyrimidines incorporation and TS inhibition, with extending infusion time to 48 hours and adding folinic acid $[10,11]$. 
Levofolinate (LF) is the active stereoisomer of folinic acid (FA), and its calcium salt (CaLF) is widely used in HDFUFA regimen, the so-called "De Gramont regimen", which currently represents the backbone of metastatic Colon Rectal Cancer (mCRC) therapy, alternatively adding oxalilplatin, irinotecan or biological agents, such as anti-EGFR or antiangiogenic monoclonal antibodies. Usually, 5FU bolus is administered at the middle of a 2-hours folinic acid infusion. Unfortunately, calcium-salt levofolinate quickly precipitates when co-administered with 5FU in continuous infusion pump, with catheter clogging and limiting its use to bolusconcomitant administration [12, 13].

As easily deduced from preclinical studies highlighted until now, prolonging simultaneous exposure of cancer cells to 5FU and folinic acid could imply a greater TS inhibition and thus a greater cell death extent. Indeed, both in cell cultures and in "vivo" animal models, only the simultaneous combination of $5 \mathrm{FU}$ and folinate salt synergistically strengthens fluorouracil cytotoxic effect [14]. Disodium LevoFolinate (NaLF) is a compound with similar pharmacological features of $\mathrm{CaLF}$, but more soluble. For this reason, NaLF can be safely administered mixed with $5 \mathrm{FU}$ in a single pump without the risk of precipitation and catheter occlusion [15]. In addition we can reach an even shorter administration time for the two drugs, requiring fewer human resources compared with sequential administration, less discomfort for patients and more compliance to treatment.

To date, no study has been made comparing the sequential standard treated with CaLF to the concomitant 5FU-NaLF regimen. Our retrospective, single-center observational study is the first with the aim of evaluating differences between these administration modalities: in particular, we wondered if co-administration of 5FU and folinic acid in continuous infusion was as effective as the classic sequential administration, or even more effective in terms of progression free- and overall survival, especially considering the aforementioned preclinical data.
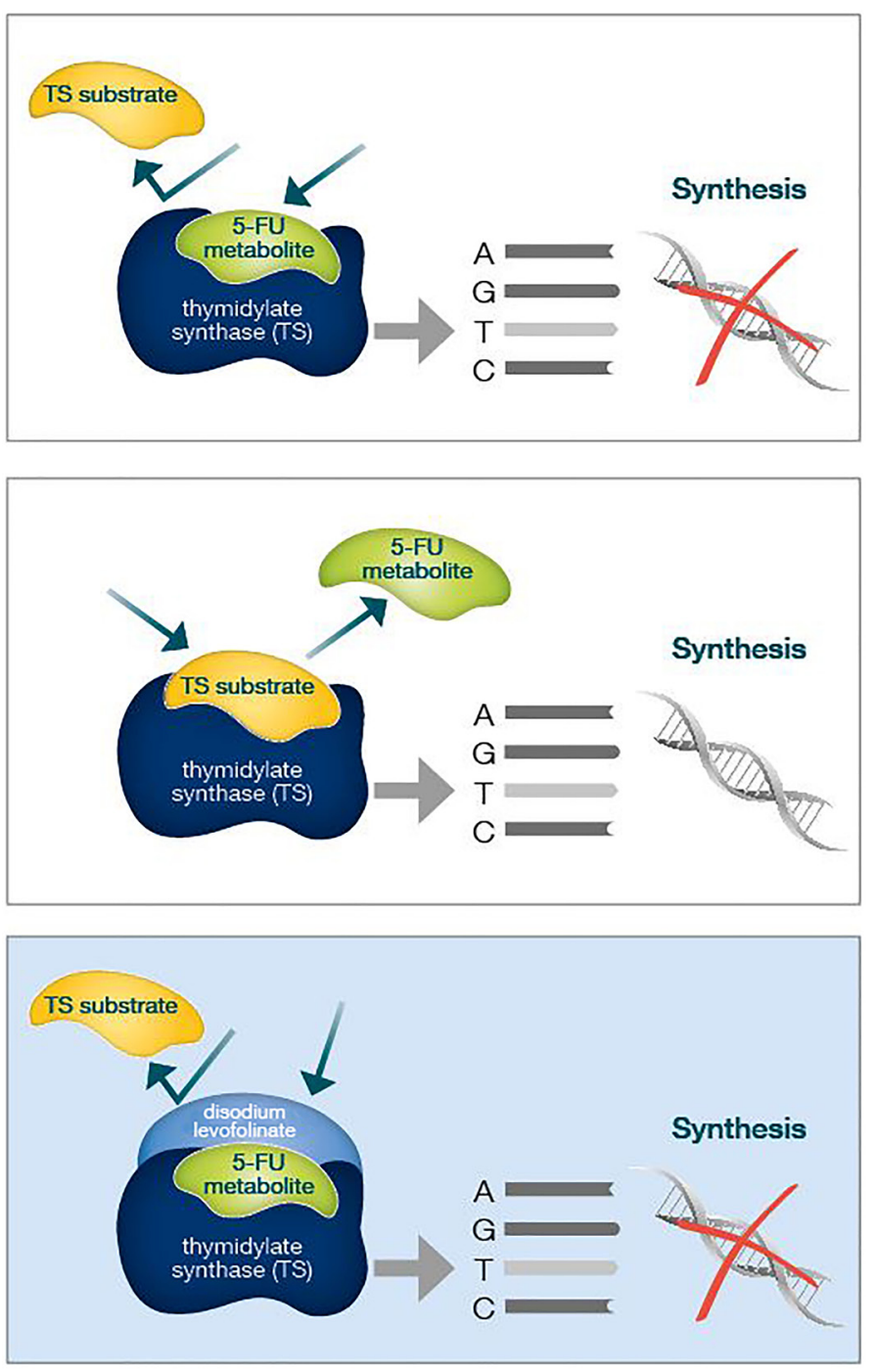

Figure 1: Folinic acid acts as a "molecular cap", strengthening the binding of 5FU to TS and therefore better inhibiting its enzymatic activity. TS overexpression in CRC cells is an acknowledged poor prognosis predicting factor. 


\section{RESULTS}

The number of patients treated with therapy based on calcium-levolinate and sodium-levolinate were 105 and 95 , respectively: between the two groups, no statistically significant difference has been found, but median follow up duration $-28,8$ for CaLF vs 18,8 months for NaLF, $p$ value 0,0001 (Mann-Whitney $U$ Test). This finding should not be surprising, because of the more recent introduction of sodium levofolinate in clinical practice. Noteworthy, in the NaLF group there was a greater frequency of RAS mutated-patients than in CaLF counterpart, although not statistically meaningful $-62 \%$ vs $50 \%, p$ value 0,074 (Table 1).

Median progression free survival of the entire study population was 14 months. Median progression free survival of patients undergoing sodium levofolinate-based therapy was significantly longer than patients treated with calcium folinate $-20,3$ versus 12,8 months, $p$ value 0,001 (Figure 2) As easily predictable, patients suffering from a $K R A S / N R A S$ mutated $\mathrm{mCRC}$ progressed earlier than wildtype counterpart, although without a clear statistically meaningful difference - 13,4 vs 16,3 months, $p$ value 0,183. KRAS/NRAS wild type NaLF treated patients progressed 9 months later than CaLF treated counterpart, though not in a statistically significant way (median PFS 23,1 vs 14 months, $p$ value 0,085); conversely, KRAS/ NRAS mutated NaLF treated patients progressed 4.7 months later than CaLF treated patients (median PFS 15.7 vs 11 months, $p$ value 0,003) (Figures 3 and 4).

In Cox multivariate analysis for PFS, NaLF halved the risk of progression - HR 0,5 - $p$ value 0,0002: the only other significant factor in Cox multivariate was chemotherapy scheme: both FOLFIRI and FOLFOX decreased progression risk compared to HD-FUFA only therapy (Table 2).

At Kaplan univariate analysis, median overall survival of entire population was 34,6 months; OS was significantly longer for KRAS/NRAS wild type patients compared to mutated counterpart - median 41,9 vs 30 months, $p$ value 0,005 (Figure 5); furthermore, patients who had undergone metastases surgery achieved a longer survival -115 vs 32,7 months $-p$ value 0,021 . No statistically significant difference was found in OS between $\mathrm{Na}$-folinate and $\mathrm{Ca}$-folinate groups $-37,7$ vs 33,4 months $-p$ value 0,151 (Figure 6).

Factors affecting overall survival in Cox univariate analysis were KRAS/NRAS mutational status, metastates surgery and chemotherapy scheme: noteworthy, in multivariate analysis KRAS/NRAS mutational status and surgery lost their statistical significance, whereas chemotherapy scheme preserved it and levofolinate achieved it - HR 0,59-p value 0,03 (Table 3 ).

\section{DISCUSSION}

The stock of therapeutic weapons available in $\mathrm{mCRC}$ has been progressively grown over the years, with improving both survival and patients' clinical outcome: adding oxaliplatin to De Gramont regimen increased progression free survival from 6,2 months to 9 months, although without a clear overall survival improvement [16]: capecitabine showed a comparable efficacy than infusional 5FU in combination with oxaliplatin [17]. With the introduction of so-called molecular target drugs into clinical practice, further improvements have been achieved in terms of survival: adding bevacizumab, the first-in-class

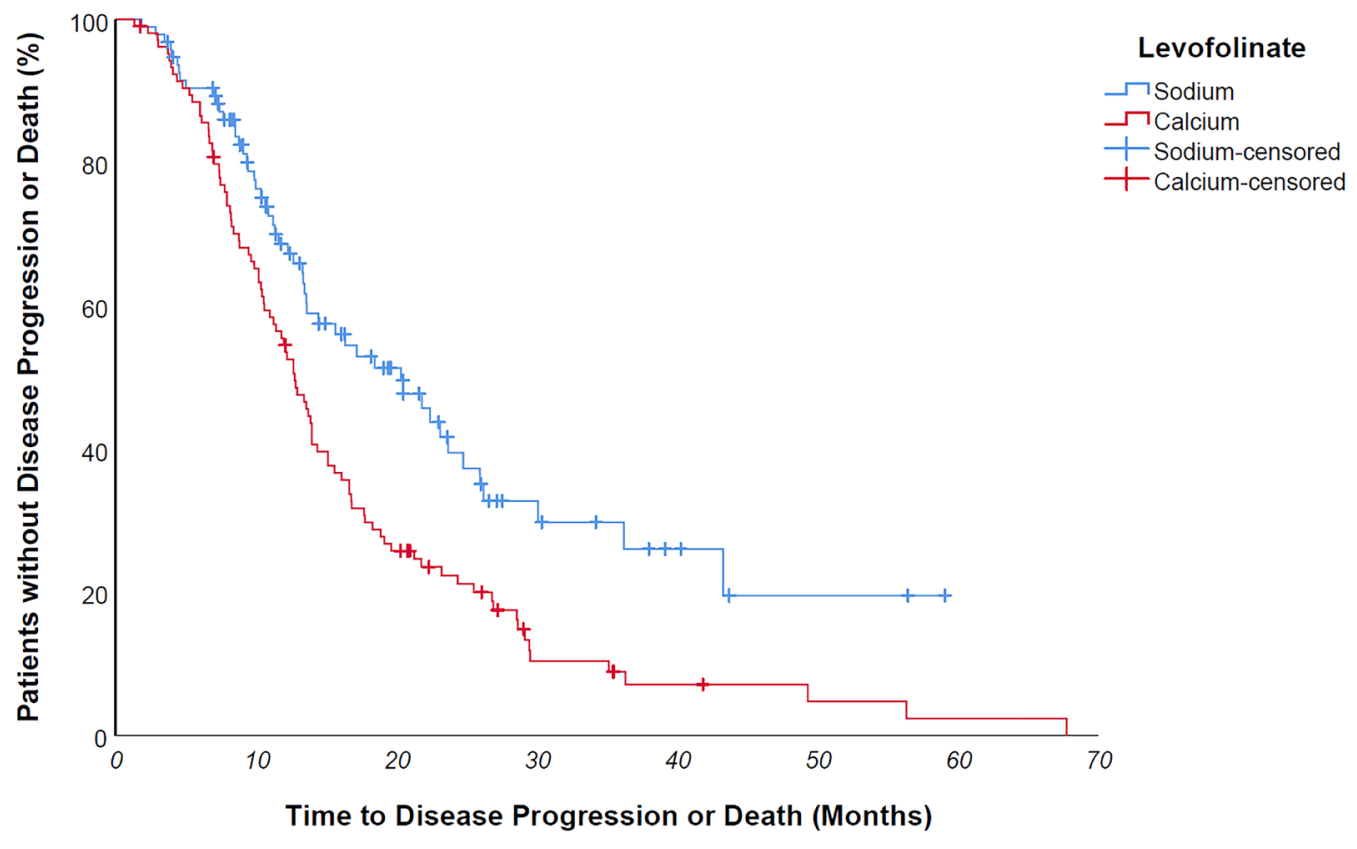

Figure 2: Median progression free survival of patients undergoing sodium levofolinate-based therapy was significantly longer than patients treated with calcium folinate $-20,3$ versus 12,8 months, $p$ value 0,001 . 
Table 1: Between the two study groups, no statistically significant difference has been found, but median follow up duration - 28,8 for CaLF vs 18,8 months for NaLF, $p$ value 0,0001 (MannWhitney $U$ Test)

\begin{tabular}{|c|c|c|c|c|c|}
\hline & \multicolumn{2}{|c|}{$\begin{array}{c}\text { Sodium } \\
95 \mathrm{pts}\end{array}$} & \multicolumn{2}{|c|}{$\begin{array}{c}\text { Calcium } \\
105 \text { pts }\end{array}$} & \multirow[t]{2}{*}{$p$ value } \\
\hline & $\mathrm{N}^{\circ}$ & $\%$ & $\mathrm{~N}^{\circ}$ & $\%$ & \\
\hline Male & 60 & 63 & 60 & 57 & $0.386^{*}$ \\
\hline Female & 35 & 37 & 45 & 43 & \\
\hline Age at first line (median) & \multicolumn{2}{|c|}{$65,6 \mathrm{yrs}$} & \multicolumn{2}{|c|}{$65,1 \mathrm{yrs}$} & $0.878^{* *}$ \\
\hline Left colon cancer & 63 & 66 & 66 & 63 & $0.610^{*}$ \\
\hline Right colon cancer & 32 & 34 & 39 & 37 & \\
\hline ALL RAS Wild Type & 36 & 38 & 53 & 50 & $0.074^{*}$ \\
\hline KRAS/NRAS Mutated & 59 & 62 & 52 & 50 & \\
\hline Metastases Surgery & & & & & \\
\hline No & 72 & 76 & 83 & 79 & $0.699^{*}$ \\
\hline Yes & 23 & 24 & 23 & 21 & \\
\hline \multicolumn{6}{|l|}{ Chemotherapy scheme } \\
\hline HD-FUFA & 7 & 8 & 2 & 2 & $0.094^{*}$ \\
\hline FOLFOX & 63 & 66 & 81 & 77 & \\
\hline FOLFIRI & 25 & 26 & 22 & 21 & \\
\hline No biological agent & 3 & 3 & 7 & 7 & $0.066^{*}$ \\
\hline Anti EGFR Mab & 29 & 31 & 45 & 43 & \\
\hline Anti VEGF Mab & 63 & 66 & 53 & 50 & \\
\hline Follow up duration (median) & \multicolumn{2}{|c|}{18,8 months } & \multicolumn{2}{|c|}{28,8 months } & $0.0001^{* *}$ \\
\hline
\end{tabular}

${ }^{* *}$ Mann-Whitney $U$ Test, "Pearson $\chi^{2}$ Test. This finding should not be surprising, because of the more recent introduction of sodium levofolinate in clinical practice. Noteworthy, in the NaLF group there was a greater frequency of RAS mutatedpatients than in CaLF counterpart, although not statistically meaningful - $62 \%$ vs $50 \%, p$ value 0,074 .

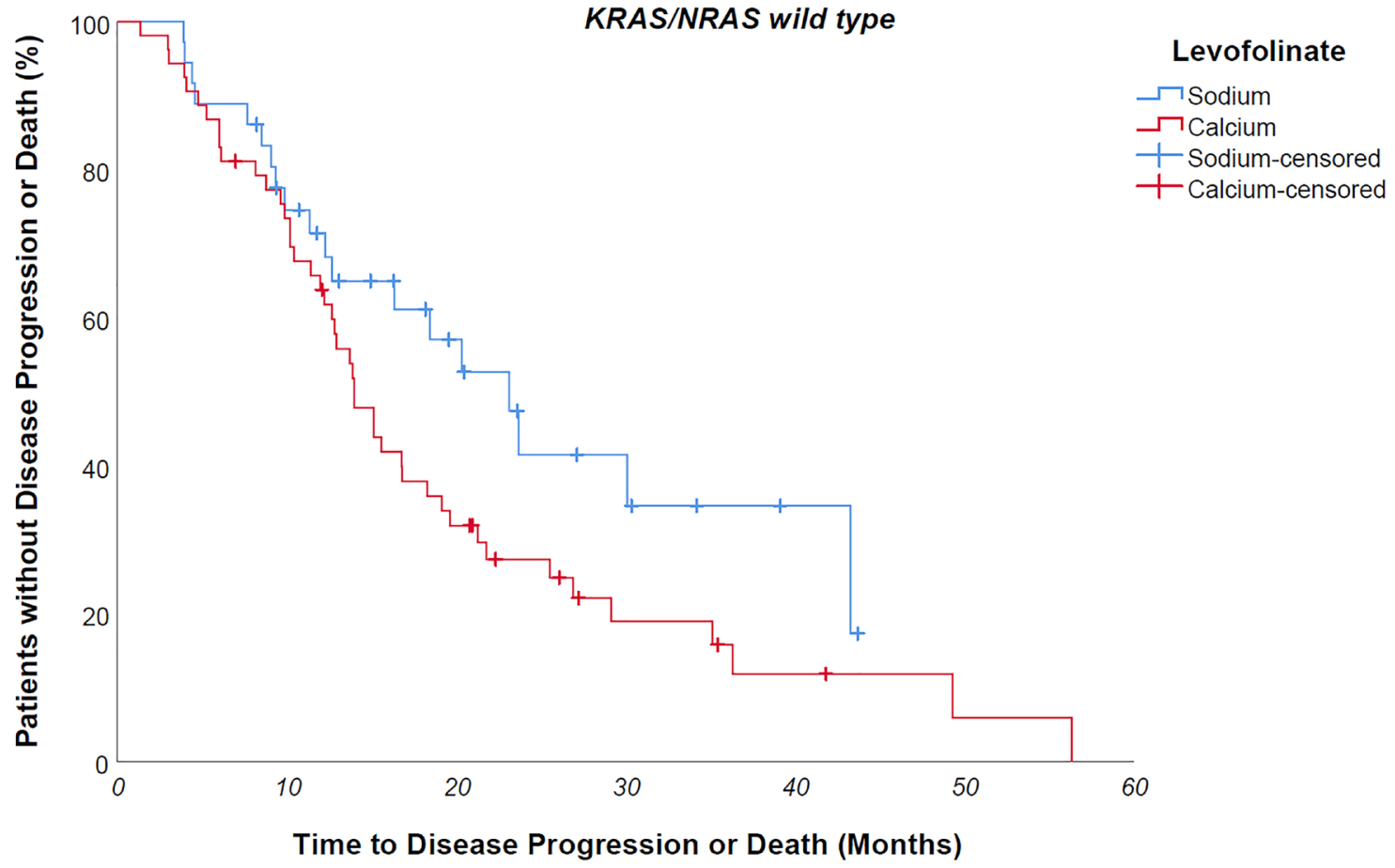

Figure 3: KRAS/NRAS wild type NaLF treated patients progressed 9 months later than CaLF treated counterpart, though not in a statistically significant way (median PFS 23,1 vs 14 months, $p$ value 0,085 ). 
Table 2: PFS Cox univariate and multivariate

\begin{tabular}{lcccccc}
\hline \multirow{2}{*}{ Cox model } & \multicolumn{3}{c}{ Univariate } & \multicolumn{3}{c}{ Multivariate } \\
\cline { 2 - 7 } & HR & CI 95\% & $\boldsymbol{p}$ value & HR & CI 95\% & $\boldsymbol{p}$ value \\
\hline Gender (M vs F) & 1.016 & $0.724-1,426$ & 0.927 & 1.042 & $0,730-1,486$ & 0.82 \\
Age at first line(continuous) & 1.002 & $0.985-1.019$ & 0.809 & 0.995 & $0,978-1,013$ & 0.59 \\
Tumor side (left colon vs right colon) & 0,733 & $0.517-1.04$ & 0.082 & 0.774 & $0,521-1,148$ & 0.2 \\
ALL RAS mutational status (WT vs mutated) & 0.799 & $0.573-1.113$ & 0.184 & 0.959 & $0,470-1,957$ & 0.9 \\
Metastases Surgery (Yes vs No) & 0.940 & $0.630-1.403$ & 0.762 & 0.978 & $0,639-1,498$ & 0.92 \\
Chemotherapy scheme & & & $\mathbf{0 . 0 1}$ & & & $\mathbf{0 . 0 0 2}$ \\
FOLFOX vs HD-FUFA & $\mathbf{0 , 3 8 0}$ & $\mathbf{0 , 1 8 4 - 0 , 7 8 7}$ & $\mathbf{0 . 0 0 9}$ & $\mathbf{0 . 2 6 6}$ & $\mathbf{0 , 1 1 9 - 0 , 5 9 5}$ & $\mathbf{0 . 0 0 1}$ \\
FOLFIRI vs HD-FUFA & $\mathbf{0 , 2 8 8}$ & $\mathbf{0 , 1 2 9 - 0 , 6 4 3}$ & $\mathbf{0 . 0 0 2}$ & $\mathbf{0 . 2 1 6}$ & $\mathbf{0 , 0 9 1 - 0 , 5 1 3}$ & $\mathbf{0 . 0 0 0 5}$ \\
Target Therapy & & & 0.370 & & & 0.84 \\
Anti EGFR vs no biological agent & 0.818 & $0.370-1.809$ & 0.620 & 0.936 & $0,369-2,375$ & 0.89 \\
$\quad$ Anti VEGF vs no biological agent & 1.049 & $0.482-2.281$ & 0.905 & 1.165 & $0,507-2,678$ & 0.72 \\
Levofolinate (Sodium vs Calcium) & $\mathbf{0 . 5 6 4}$ & $\mathbf{0 . 4 0 1 - 0 . 7 9 5}$ & $\mathbf{0 . 0 0 1}$ & $\mathbf{0 . 5}$ & $\mathbf{0 , 3 4 7 - 0 , 7 2 0}$ & $\mathbf{0 . 0 0 0 2}$ \\
\hline
\end{tabular}

In Cox multivariate analysis for PFS, NaLF halved the risk of progression - HR 0,5 - $p$ value 0,0002 : the only other significant factor in Cox multivariate was chemotherapy scheme: both FOLFIRI and FOLFOX decreased progression risk compared to HD-FUFA only therapy.

\section{Table 3: OS Cox univariate and multivariate}

\begin{tabular}{lcccccc}
\hline Cox model & \multicolumn{3}{c}{ Univariate } & \multicolumn{3}{c}{ Multivariate } \\
\cline { 2 - 7 } & HR & CI 95\% & $\boldsymbol{p}$ value & HR & CI 95\% & $\boldsymbol{p}$ value \\
\hline Gender (M vs F) & 1.13 & $0,745-1,715$ & 0.565 & 1.101 & $0,713-1,700$ & 0.665 \\
Age at first line(continuous) & 1.013 & $0.985-1.019$ & 0.809 & 1.006 & $0,985-1,027$ & 0.596 \\
Tumor side (left colon vs right colon) & 1.063 & $0.701-1.614$ & 0.773 & 1,050 & $0,666-1,653$ & 0.834 \\
ALL RAS mutational status (WT vs mutated) & $\mathbf{0 . 5 6}$ & $\mathbf{0 , 3 7 0 - 0 , 8 4 7}$ & $\mathbf{0 . 0 0 6}$ & 0.731 & $0,298-1,794$ & 0.494 \\
Metastases Surgery (Yes vs No) & $\mathbf{0 . 5 1 8}$ & $\mathbf{0 . 2 9 4 - 0 . 9 1 4}$ & $\mathbf{0 . 0 2 3}$ & 0.58 & $0,321-1,048$ & 0.071 \\
Chemotherapy scheme & & & $\mathbf{0 . 0 0 3}$ & & & $\mathbf{0 . 0 0 4}$ \\
FOLFOX vs HD-FUFA & $\mathbf{0 , 3 2 7}$ & $\mathbf{0 , 1 3 1 - 0 , 8 1 7}$ & $\mathbf{0 . 0 1 7}$ & $\mathbf{0 . 3 5 0}$ & $\mathbf{0 , 1 2 7 - 0 , 9 6 3}$ & $\mathbf{0 . 0 4 2}$ \\
FOLFIRI vs HD-FUFA & $\mathbf{0 , 1 6 7}$ & $\mathbf{0 , 0 5 9 - 0 , 4 7 2}$ & $\mathbf{0 . 0 0 1}$ & $\mathbf{0 . 1 6 7}$ & $\mathbf{0 , 0 5 4 - 0 , 5 1 5}$ & $\mathbf{0 . 0 0 2}$ \\
Target Therapy & & & 0.054 & & & 0.721 \\
Anti EGFR vs no biological agent & 0.716 & $0.297-1.722$ & 0.455 & 0.905 & $0,305-2,682$ & 0.857 \\
Anti VEGF vs no biological agent & 1.223 & $0.526-2.843$ & 0.640 & 1.300 & $0,521-3,243$ & 0.573 \\
Levofolinate (Sodium vs Calcium) & 0.718 & $0.456-1.130$ & 0.152 & $\mathbf{0 . 5 8 5}$ & $\mathbf{0 , 3 6 0 - 0 , 9 5 1}$ & $\mathbf{0 . 0 3 1}$ \\
\hline
\end{tabular}

Factors affecting overall survival in Cox univariate analysis were ALL RAS mutational status, metastates surgery and chemotherapy scheme: noteworthy, in multivariate analysis ALL RAS and surgery lost their statistical significance, whereas chemotherapy scheme preserved it and levofolinate achieved it - HR 0,59- $p$ value 0,03 .

antiangiogenic agent, to 5FU-FA based therapy, resulted in 10,6 median PFS versus the 6,2 months of no bevacizumab counterpart [18]. Afterwards, Saltz et al. demonstrated that the addition of bevacizumab to oxaliplatin-based chemotherapy regimens significantly improves PFS (11.1 vs 8.6 months), irrespective of RAS mutational status [19].

On the other hand, anti-EGFR agents improved PFS in KRAS wild type tumor affected patients: cetuximab in combination with FOLFIRI and panitumumab with FOLFOX led to an increase of PFS up to 9,9 and 10 months, respectively. FOLFIRI-cetuximab showed a PFS of 10.9 months in the KRAS/BRAF wild type subgroup, although without determining a statistically significant difference in PFS compared to the FOLFIRI-only treated group due to the low sample size $[20,21]$.

In our center experience, median PFS of patients was 12,8 months, slightly longer than previously reported: this discrepancy, although minimal, could be explained by the quite large percentage of patients who underwent surgery.

Increased survival for patients undergoing NaLF based therapy could be the consequence of a greater and more effective TS inhibition. A plausible reason for ALL RAS absence of significance on OS multivariate compared to univariate analysis can be found in extremely wide expression of TS in colon cancer cells. Indeed, on 
one hand we have an oncogene addiction toward EGFR pathway in ALL RAS wild type cells, but on the other hand we know that TS is essential for non-oncogenic pathways of cancer cells regardless of EGFR or RAS activation. Thus, we could postulate that the greater inhibition of TS we achieve with 5FU-LF combination, the lesser the role for the EGFR-driven oncogenic pathway. Noteworthy, there are coincidently more RAS mutated patients in NaLF group than the CaLF counterpart. Furthermore, as already shown, follow-up is shorter for

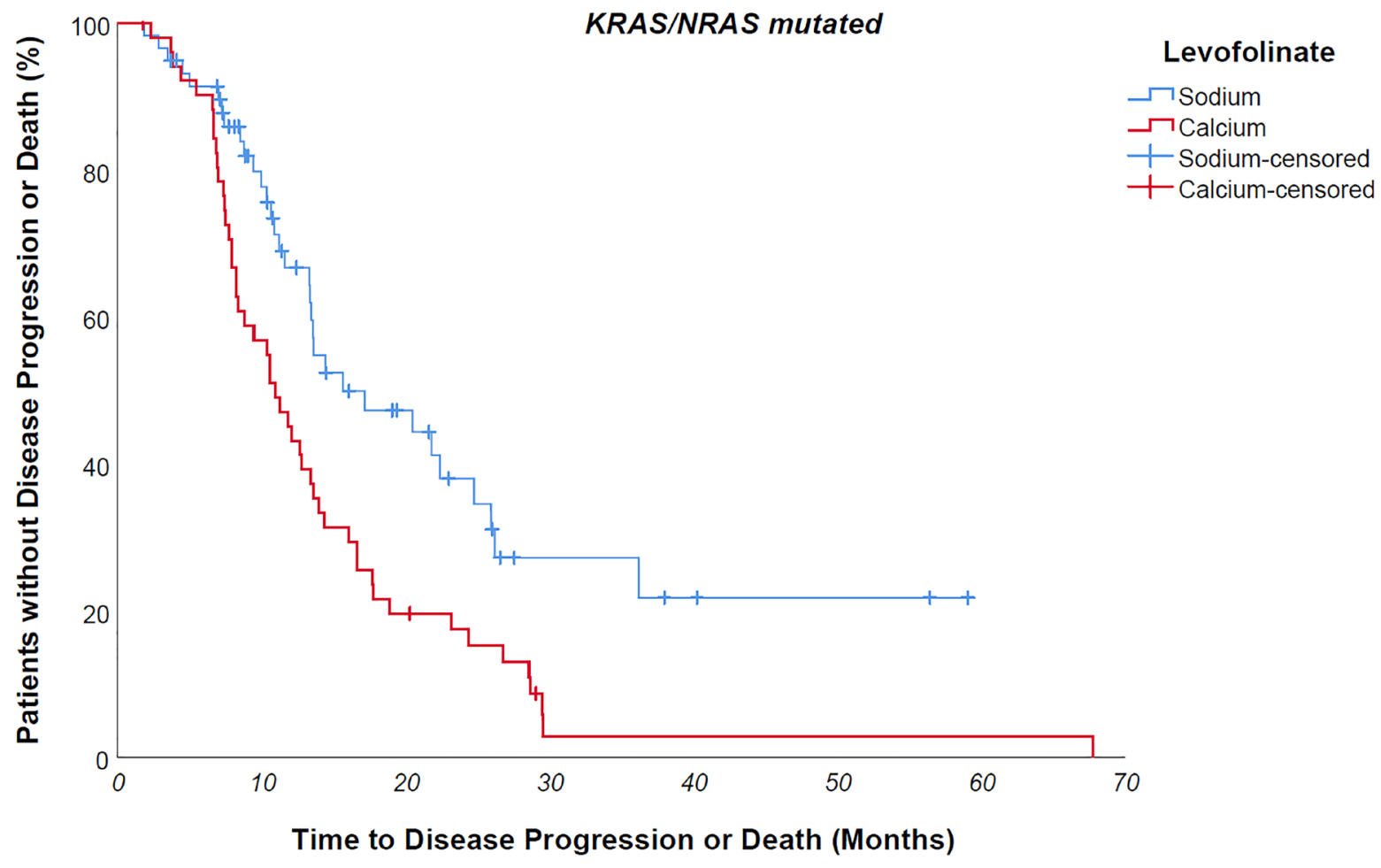

Figure 4: KRAS/NRAS mutated NaLF treated patients progressed 4.7 months later than CaLF treated patients (median PFS 15.7 vs 11 months, $p$ value 0,003).

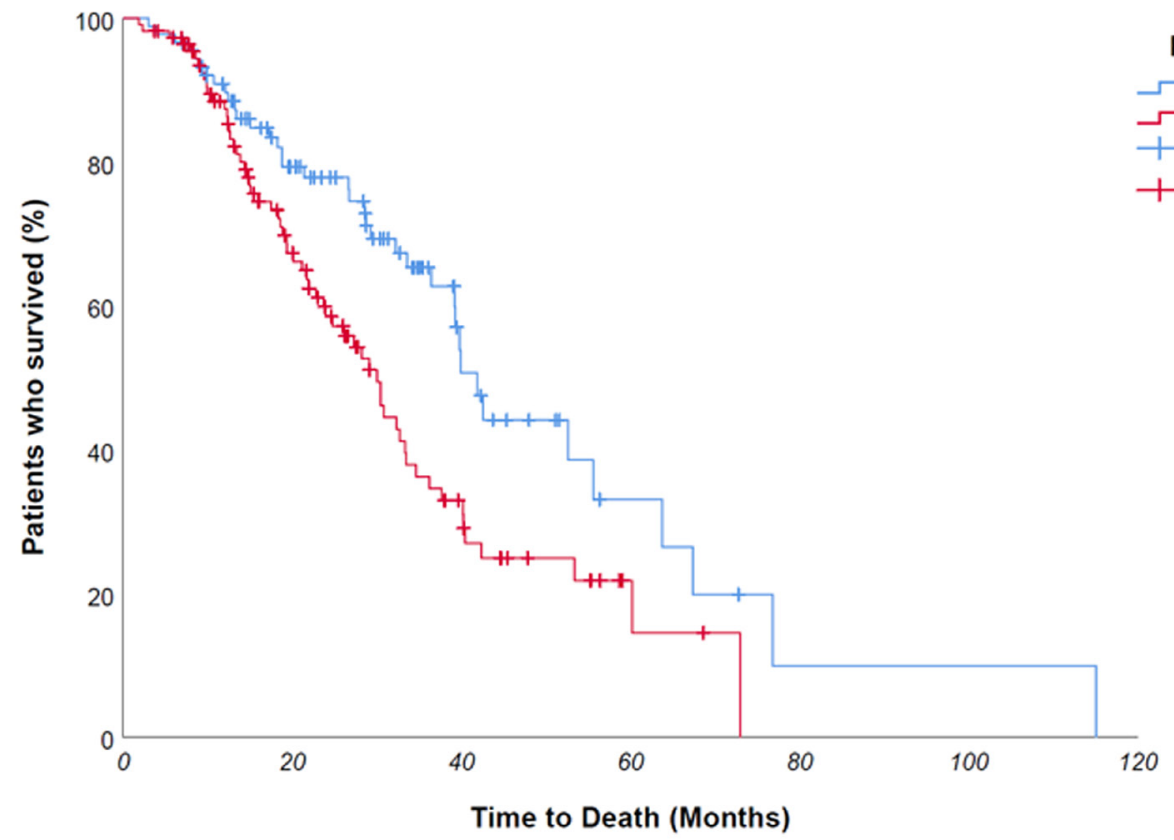

RAS mutational status

$\rightarrow$ ALL RAS Wild Type $\neg$ KRAS/NRAS mutated - ALL RAS Wild Type-censored + KRAS/NRAS mutatedcensored

Figure 5: At Kaplan univariate analysis, OS was significantly longer for ALL RAS wild type patients compared to mutated counterpart - median 41,9 vs 30 months, $p$ value 0,005 . 
NaLF-treated group, with fewer death events: probably, as events increase this analysis could become significant. Finally, we must highlight the therapy switch over of a large number of patients who underwent NaLF-based therapy in second line after first-line CaLF-based therapy, partially disguising the overall survival difference between the two groups.

\section{MATERIALS AND METHODS}

This mono-institutional, observational study retrospectively reviewed medical records of 200 patients suffering from mCRC who underwent first-line therapy: patients did not provide any written informed consent, in accordance with the Declaration of Helsinki and Italian Privacy Protection Commissioner. All patients underwent 5-fluorouracil based chemotherapy: HDFUFA (5FU bolus $400 \mathrm{mg} / \mathrm{m}^{2}$, calcium levofolinate 200 $\mathrm{mg} / \mathrm{m}^{2}, 5 F U 48$ hours-continuous infusion $2400 \mathrm{mg} / \mathrm{m}^{2}$ ) intravenous every 2 weeks, FOLFOX6 (5FU bolus 400 $\mathrm{mg} / \mathrm{m}^{2}$, calcium levofolinate $200 \mathrm{mg} / \mathrm{m}^{2}, 5 F U 48$ hourscontinuous infusion $2400 \mathrm{mg} / \mathrm{m}^{2}$ and Oxaliplatin 85 $\mathrm{mg} / 2$ ) intravenous every 2 weeks, FOLFIRI (5FU bolus $400 \mathrm{mg} / \mathrm{m}^{2}$, calcium levofolinate $200 \mathrm{mg} / \mathrm{m}^{2}$, 5FU 48 hours-continuous infusion $2400 \mathrm{mg} / \mathrm{m}^{2}$ and Irinotecan $180 \mathrm{mg} / 2$ ) intravenous every 2 weeks. When sodium levofolinate was used, it was co-administered along with 5FU continuous infusion, and 5FU bolus was administered just before elastomeric pump application; on the other hand, 5FU bolus was administered halfway through 2 hours calcium levofolinate infusion. Biological agents (antiangiogenic - bevacizumab or aflibercept, and antiEGFR - panitumumab or cetuximab) were added to chemotherapy backbone depending on ALL RAS mutation status or clinical conditions by clinician choice.

Primary endpoints were Progression Free Survival (PFS) and Overall Survival (OS): PFS was defined as the interval between the first therapy administration and the date of disease progression or death for any cause; disease progression was defined as radiological tumor progression according to Response Evaluation Criteria in Solid Tumors, RECIST, version 1.1, or clinical progression, including death; OS was defined as the time from firstline therapy start to death from any cause.

Patients' demographic and baseline characteristics, treatment patterns and acknowledged prognostic factors have been collected, with categorical variables being described by patient counts and percentages. Data were summarized as frequencies for categorical variables and mean and range values for continuous variables: Pearson chi-square test and Mann-Whitney Test for independent samples were used to compare such variables among groups, respectively.

Univariate analysis for median progression freeand overall survival was performed by Kaplan-Meier estimator: PFS and OS curves were obtained, and selected variables were compared using two-sided log-rank test. Hazard ratios (HR) were calculated by Cox Regression univariate and multivariate analysis: a $p$ value $\leq 0.05$ was considered statistically significant. The SPSS statistical package version 26.0 (SPSS Inc., Chicago, IL) was used for all statistical analysis.

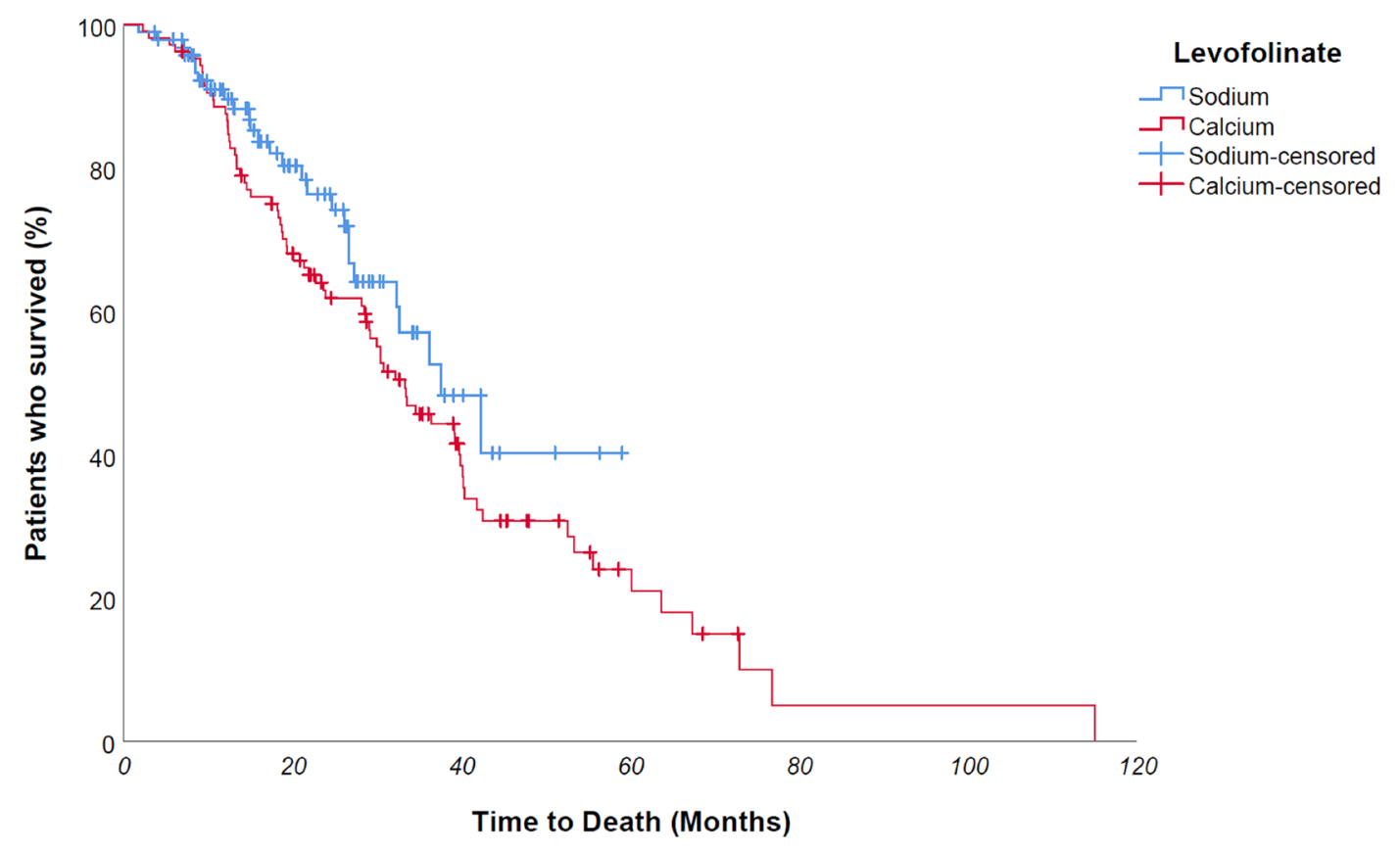

Figure 6: No difference was found in univariate OS analysis for folinic salt use - 37,7 vs 33,4 months - $p$ value 0,151 maybe due to therapy switch over of a large number of patients who underwent NaLF-based therapy in second line after first-line CaLF-based therapy, partially disguising the overall survival difference between the two groups. 


\section{CONCLUSIONS}

Finally, in our experience concomitant administration of $5 \mathrm{FU}$ and sodium levofolinate prolongs the time to disease progression of patients suffering from $\mathrm{mCRC}$, moreover decreasing death risk by $40 \%$. Further prospective, headto-head trials are warranted in order to confirm these findings, although in our retrospective experience the use of sodium levofolinate showed a clear clinical benefit for patients, regardless of RAS mutational status, tumor side or biological agent added to chemotherapy.

\section{Author contributions}

Francesco Jacopo Romano conceived the study, performed statistical analysis, drafted and revised the manuscript. Carmela Barbato, Vincenzo Di Lauro, Roberto Fiorentino, Francesca Ambrosio, Maresa Cammarota, Bruno Chiurazzi, Livio Puglia, Sarah Scagliarini, Raffaella Ruocco, Maria Fiorella Brangi, Carmen Mocerino and Ivana Cerillo contributed to data collection and manuscript revision. Dario Arundine and Maria Biglietto contributed to data collection, manuscript drafting and revision. Ferdinando Riccardi contibuted to study concept, manuscript drafting and critical revision.

\section{CONFLICTS OF INTEREST}

Authors have no conflicts of interest to declare.

\section{REFERENCES}

1. Kubota T, Watanabe M, Otani Y, Kitajima M, Fukushiuma M. Different pathways of 5-fluorouracil metabolism after continuous venous or bolus injection in patients with colon carcinoma: possible predictive value of thymidylate synthetase mRNA and ribonucleotide reductase for 5-fluorouracil sensitivity. Anticancer Res. 2002; 22:3537-3540. [PubMed]

2. Thrall MM, Wood P, King V, Rivera W, Hrushesky W. Investigation of the comparative toxicity of 5-FU bolus versus 5-FU continuous infusion circadian chemotherapy with concurrent radiation therapy in locally advanced rectal cancer. Int J Radiat Oncol Biol Phys. 2000; 46:873-881. https://doi.org/10.1016/s0360-3016(99)00456-3. [PubMed]

3. Hartmann JT, Köhne CH, Schmoll HJ, Daikeler T, Kanz L, Bokemeyer C. Is continuous 24-hour infusion of 5-fluorouracil plus high-dose folinic acid effective in patients with progressive or recurrent colorectal cancer? A phase II study. Oncology. 1998; 55:320-325. https://doi. org/10.1159/000011869. [PubMed]

4. Hartung G, Hofheinz RD, Wein A, Riedel C, Rost A, Fritze D, Kreuser ED, Drees M, Kühnel J, Hehlmann R, Queisser W. Phase II study of a weekly 24-hour infusion with 5-fluorouracil and simultaneous sodium-folinic acid in the first-line treatment of metastatic colorectal cancer. Onkologie. 2001; 24:457-462. https://doi. org/10.1159/000055126. [PubMed]

5. Aschele C, Lonardi S, Monfardini S. Thymidylate Synthase expression as a predictor of clinical response to fluoropyrimidine-based chemotherapy in advanced colorectal cancer. Cancer Treat Rev. 2002; 28:27-47. https://doi.org/10.1053/ctrv.2002.0253. [PubMed]

6. Popat S, Matakidou A, Houlston RS. Thymidylate synthase expression and prognosis in colorectal cancer: a systematic review and meta-analysis. J Clin Oncol. 2004; 22:529-536. https://doi.org/10.1200/JCO.2004.05.064. [PubMed]

7. Moran RG, Keyomarsi K. Biochemical rationale for the synergism of 5-fluorouracil and folinic acid. NCI Monogr. 1987; 5:159-163. [PubMed]

8. Spears CP, Gustavsson BG, Frösing R. Folinic acid modulation of fluorouracil: tissue kinetics of bolus administration. Invest New Drugs. 1989; 7:27-36. https:// doi.org/10.1007/BF00178189. [PubMed]

9. Noordhuis P, Holwerda U, Van der Wilt CL, Van Groeningen CJ, Smid K, Meijer S, Pinedo HM, Peters GJ. 5-Fluorouracil incorporation into RNA and DNA in relation to thymidylate synthase inhibition of human colorectal cancers. Ann Oncol. 2004; 15:1025-1032. https://doi. org/10.1093/annonc/mdh264. [PubMed]

10. De Gramont A, Krulik M, Cady J, Lagadec B, Maisani JE, Loiseau JP, Grange JD, Gonzalez-Canali G, Demuynck B, Louvet C. High-dose folinic acid and 5-fluorouracil bolus and continuous infusion in advanced colorectal cancer. Eur J Cancer Clin Oncol. 1988; 24:1499-1503. https://doi. org/10.1016/0277-5379(88)90341-0. [PubMed]

11. De Gramont A, Louvet C, Krulik M. The French experience with infusional 5-fluorouracil in advanced colorectal cancer. J Infus Chemother. 1996; 6:133-136. [PubMed]

12. Ardalan B, Chua L, Tian EM, Reddy R, Sridhar K, Benedetto P, Richman S, Legaspi A, Waldman S, Morrell L. A phase II study of weekly 24-hour infusion with highdose fluorouracil with leucovorin in colorectal carcinoma. J Clin Oncol. 1991; 9:625-630. https://doi.org/10.1200/ JCO.1991.9.4.625. [PubMed]

13. Bruch HR, Esser M. Catheter occlusion by calcium carbonate during simultaneous infusion of 5-FU and calcium folinate. Onkologie. 2003; 26:469-472. https://doi. org/10.1159/000072981. [PubMed]

14. Di Paolo A, Orlandi P, Di Desidero T, Danesi R, Bocci G. Simultaneous, But Not Consecutive, Combination with Folinate Salts Potentiates 5-Fluorouracil Antitumor Activity In Vitro and In Vivo. Oncol Res. 2017; 25:1129-1140. https:// doi.org/10.3727/096504017X14841698396900. [PubMed]

15. Ratti M, Hahne JC, Toppo L, Castelli E, Petrelli F, Passalacqua R, Barni S, Tomasello G, Ghidini M. Major innovations and clinical applications of disodiumlevofolinate: a review of available preclinical and clinical data. Ther Adv Med Oncol. 2019; 11:1758835919853954. https://doi.org/10.1177/1758835919853954. [PubMed] 
16. de Gramont A, Figer A, Seymour M, Homerin M, Hmissi A, Cassidy J, Boni C, Cortes-Funes H, Cervantes A, Freyer G, Papamichael D, Le Bail N, Louvet C, et al. Leucovorin and fluorouracil with or without oxaliplatin as first-line treatment in advanced colorectal cancer. J Clin Oncol. 2000; 18:2938-47. https://doi.org/10.1200/JCO.2000.18.16.2938. [PubMed]

17. Cassidy J, Clarke S, Díaz-Rubio E, Scheithauer W, Figer A, Wong R, Koski S, Lichinitser M, Yang TS, Rivera F, Couture F, Sirzén F, Saltz L. Randomized phase III study of capecitabine plus oxaliplatin compared with fluorouracil/ folinic acid plus oxaliplatin as first-line therapy for metastatic colorectal cancer. J Clin Oncol. 2008; 26:200612. https://doi.org/10.1200/JCO.2007.14.9898. [PubMed]

18. Hurwitz HI, Fehrenbacher L, Hainsworth JD, Heim W, Berlin J, Holmgren E, Hambleton J, Novotny WF, Kabbinavar F. Bevacizumab in combination with fluorouracil and leucovorin: an active regimen for first-line metastatic colorectal cancer. J Clin Oncol. 2005; 23:3502-8. https://doi.org/10.1200/JCO.2005.10.017. [PubMed]

19. Saltz L, Clarke S, Diaz-Rubio E, Scheithauer W, Figer A, Wong R, Koski S, Lichinitser M, Yang T, Cassidy
J. Bevacizumab (Bev) in combination with XELOX or FOLFOX4: Updated efficacy results from XELOX-1/ NO16966, a randomized phase III trial in first-line metastatic colorectal cancer. J Clin Oncol. 2007; 25:40284028. https://doi.org/10.1200/jco.2007.25.18 suppl.4028.

20. Van Cutsem E, Lang I, Folprecht G, Nowacki M, Barone C, Shchepotin I, Maurel J, Cunningham D, Celik I, Kohne C. Cetuximab plus FOLFIRI: Final data from the CRYSTAL study on the association of KRAS and BRAF biomarker status with treatment outcome. J Clin Oncol. 2010; 28:3570-3570. https://doi.org/10.1200/jco.2010.28.15 suppl.3570.

21. Douillard JY, Siena S, Cassidy J, Tabernero J, Burkes R, Barugel M, Humblet Y, Bodoky G, Cunningham D, Jassem J, Rivera F, Kocákova I, Ruff P, et al. Final results from PRIME: randomized phase III study of panitumumab with FOLFOX4 for first-line treatment of metastatic colorectal cancer. Ann Oncol. 2014; 25:1346-1355. https://doi. org/10.1093/annonc/mdu141. [PubMed] 